\title{
Injury To Carotid Artery, CTCAE
}

National Cancer Institute

\section{Source}

National Cancer Institute. Injury To Carotid Artery, CT CAE. NCI Thesaurus. Code C143586.

A finding of damage to the carotid artery. 